\title{
Unparticle Example in 2D
}

\section{Citation}

Georgi, Howard, Yevgeny Kats. 2008. Unparticle Example in 2D. Physical Review Letters 101, 131603.

\section{Published Version}

http://dx.doi.org/10.1103/PhysRevLett.101.131603

\section{Permanent link}

http://nrs.harvard.edu/urn-3:HUL.InstRepos:2794951

\section{Terms of Use}

This article was downloaded from Harvard University's DASH repository, and is made available under the terms and conditions applicable to Open Access Policy Articles, as set forth at http:// nrs.harvard.edu/urn-3:HUL.InstRepos:dash.current.terms-of-use\#OAP

\section{Share Your Story}

The Harvard community has made this article openly available.

Please share how this access benefits you. Submit a story.

Accessibility 


\title{
Unparticle Example in 2D
}

\author{
Howard Georgi* and Yevgeny Kats ${ }^{+}$ \\ Center for the Fundamental Laws of Nature, Jefferson Physical Laboratory, Harvard University, \\ Cambridge, Massachusetts 02138, USA
}

(Received 27 July 2008; published 25 September 2008)

\begin{abstract}
We discuss what can be learned about unparticle physics by studying simple quantum field theories in one space and one time dimension. We argue that the exactly soluble $2 \mathrm{D}$ theory of a massless fermion coupled to a massive vector boson, the Sommerfield model, is an interesting analog of a Banks-Zaks model, approaching a free theory at high energies and a scale-invariant theory with nontrivial anomalous dimensions at low energies. We construct a toy standard model coupling to the fermions in the Sommerfield model and study how the transition from unparticle behavior at low energies to free particle behavior at high energies manifests itself in interactions with the toy standard model particles.
\end{abstract}

The term "unparticle physics" was coined by one of us to describe a situation in which standard model physics is weakly coupled at high energies to a sector that flows to a scale-invariant theory in the infrared [1,2]. In this class of models, one may see surprising effects from the production of unparticle stuff [3] in the scattering of standard model particles. Studying such models forces us to confront some interesting issues in scale-invariant theories and effective field theories.

It is important to remember that unparticle physics is not just about a scale-invariant theory. There are two other important ingredients. A crucial one is the coupling of the unparticle fields to the standard model. Without this coupling, we would not be able to "see" unparticle stuff. Also important is the transition in the Banks-Zaks theory [4] from which unparticle physics emerges from perturbative physics at high energies to scale-invariant unparticle behavior at low energies. This allows us to find wellcontrolled perturbative physics that produces the coupling of the unparticle sector to the standard model. Without this transition, the coupling of the standard model to the unparticle fields would have to be put in by hand in a completely arbitrary way, and much of the phenomenological interest of the unparticle metaphor would be lost.

In this Letter, we explore the physics of the transition from unparticle behavior at low energies to perturbative behavior at high energies in a model with one space and one time dimension in which the analog of the Banks-Zaks model is exactly solvable. This will enable us to see how the transition takes place explicitly in a simple inclusive scattering process (Fig. 1).

We begin by describing our analog Banks-Zaks model and its solution. It is a 2D model of massless fermions coupled to a massive vector field. We call it the Sommerfield model because it was solved by Sommerfield [5] in 1963 [6]. Next, we describe the high-energy physics that couples the Sommerfield model to our toy standard model, which is simply a massive scalar carrying a global
$U(1)$ charge. In the infrared, the resulting interaction flows to a coupling of two charged scalars to an unparticle field with a nontrivial anomalous dimension. We apply the operator product expansion to the solution of the Sommerfield model to find the correlation functions of the low-energy unparticle operator. Finally, we study the simplest unparticle process shown in Fig. 1 in which two toy standard model scalars "disappear" into unparticle stuff. Because we have the exact solution for the unparticle correlation functions, we can see precisely how the system makes the transition from low-energy unparticle physics to the high-energy physics of free particles. The answer is simple and elegant. The "spectrum" of the model consists of unparticle stuff and massive bosons. As the incoming energy of the standard model scalars is increased, the unparticle stuff is always there, but more and more massive bosons are emitted, and the combination becomes more and more like the free-fermion cross section.

The Sommerfield(-Thirring-Wess) model [6,7] is the Schwinger model [8] with an additional mass term for

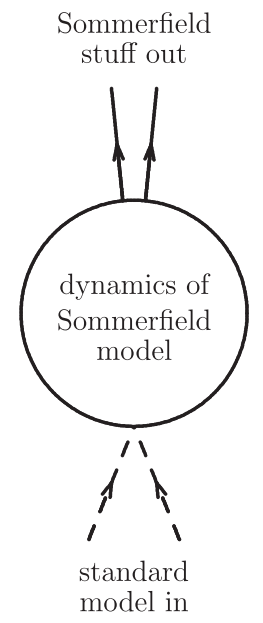

FIG. 1. A disappearance process. 
the vector boson [9]:

$$
\mathcal{L}=\bar{\psi}(i \not \supset-e \not A) \psi-\frac{1}{4} F^{\mu \nu} F_{\mu \nu}+\frac{m_{0}^{2}}{2} A^{\mu} A_{\mu} .
$$

We are interested in this theory since it is exactly solvable and (unlike the Schwinger model) has fractional anomalous dimensions. In particular, we are interested in the composite operator

$$
\mathcal{O} \equiv \psi_{2}^{*} \psi_{1}
$$

because, in the low-energy theory, it scales with an anomalous dimension.

The solution for all fermion Green's functions in the model can be written down explicitly, in terms of propagators for free fermions, and for massless and massive scalar fields with mass $m$ :

$$
m^{2}=m_{0}^{2}+\frac{e^{2}}{\pi}
$$

The physical mass $m$ plays the role in this model of the unparticle scale $\Lambda_{u}$ from Ref. [1], setting the scale of the transition between free particle behavior at high energies and unparticle behavior at low energies. Explicitly, the scalar propagators are [10]

$$
\begin{gathered}
\Delta(x)=\int \frac{d^{2} p}{(2 \pi)^{2}} \frac{e^{-i p x}}{p^{2}-m^{2}+i \epsilon} \\
=-\frac{i}{2 \pi} K_{0}\left(m \sqrt{-x^{2}+i \epsilon}\right), \\
D(x)=\int \frac{d^{2} p}{(2 \pi)^{2}} \frac{e^{-i p x}}{p^{2}+i \epsilon}=\frac{i}{4 \pi} \ln \left(\frac{-x^{2}+i \epsilon}{x_{0}^{2}}\right) .
\end{gathered}
$$

The $n$-point functions for the $\mathcal{O}$ and $\mathcal{O}^{*}$ fields can then be constructed using the operator product expansion. We will describe all of this in detail in a separate publication $[11,12]$. Here we will simply write down and use the result for the 2-point function in position space

$$
i \Delta_{\mathcal{O}}(x) \equiv\left\langle 0\left|T \mathcal{O}(x) \mathcal{O}^{*}(0)\right| 0\right\rangle=\frac{B(x)}{4 \pi^{2}\left(-x^{2}+i \epsilon\right)},
$$

where

$$
\begin{aligned}
B(x) & =\exp \left[i \frac{4 e^{2}}{m^{2}}\{[\Delta(x)-\Delta(0)]-[D(x)-D(0)]\}\right] \\
& =\exp \left[\frac{2 e^{2}}{\pi m^{2}}\left[K_{0}\left(m \sqrt{-x^{2}+i \epsilon}\right)+\ln \left(\xi m \sqrt{-x^{2}+i \epsilon}\right)\right]\right]
\end{aligned}
$$

with

$$
\xi=e^{\gamma_{E}} / 2 .
$$

In the short-distance limit $\left(\left|x^{2}\right| \ll 1 / m^{2}\right), B(x) \rightarrow 1$, and one obtains free-fermion behavior. In the large-distance limit $\left(\left|x^{2}\right| \gg 1 / m^{2}\right), K_{0}$ does not contribute, so $B(x)$ is just a power of $x^{2}$, and the 2-point function is proportional to an unparticle propagator [13]

$$
i \Delta_{\mathcal{O}}(x) \rightarrow i \Delta_{U}(x)=\frac{1}{4 \pi^{2}(\xi m)^{2 a}\left(-x^{2}+i \epsilon\right)^{1+a}},
$$

where

$$
a \equiv-\frac{e^{2}}{\pi m^{2}}=-\frac{1}{1+\pi m_{0}^{2} / e^{2}} .
$$

Thus at large distance and low energies, the composite operator $\mathcal{O}$ scales with dimension $1+a$, corresponding to an anomalous dimension of $a$ for $\psi_{2}^{*} \psi_{1}$. For $0<m_{0}<$ $\infty, a$ is fractional, which leads to unparticle behavior. In momentum space

$$
\begin{aligned}
i \Delta_{\mathcal{U}}(p) & =\frac{i A(a)}{2(\xi m)^{2 a} \sin (\pi a)}\left(-p^{2}-i \epsilon\right)^{a} \\
& =\frac{A(a)}{2 \pi(\xi m)^{2 a}} \int_{0}^{\infty} d M^{2}\left(M^{2}\right)^{a} \frac{i}{p^{2}-M^{2}+i \epsilon}
\end{aligned}
$$

where the function

$$
A(a) \equiv-\frac{\sin (\pi a) \Gamma(-a)}{2^{1+2 a} \pi \Gamma(1+a)}
$$

is positive in the range relevant to our model $(-1<a<$ $0)$. Since

$$
\operatorname{Im} \Delta_{\mathcal{U}}(p)=-\frac{A(a)}{2(\xi m)^{2 a}} \theta\left(p^{2}\right)\left(p^{2}\right)^{a},
$$

the unparticle phase space is

$$
\Phi_{u}(p)=\frac{A(a)}{(\xi m)^{2 a}} \theta\left(p^{0}\right) \theta\left(p^{2}\right)\left(p^{2}\right)^{a} .
$$

To generate a coupling to a toy standard model, we assume that the very high-energy theory includes the interaction

$$
\begin{aligned}
\mathcal{L}_{\text {int }} & =\frac{\mu}{2}\left[\bar{\psi}\left(1+\gamma^{5}\right) \chi \phi^{*}+\bar{\psi}\left(1-\gamma^{5}\right) \chi \phi\right]+\text { H.c. } \\
& =\mu\left(\psi_{2}^{*} \chi_{1} \phi^{*}+\psi_{1}^{*} \chi_{2} \phi\right)+\text { H.c. }
\end{aligned}
$$

that couples the fermion $\psi$ of the unparticle sector to a neutral complex scalar $\phi$ with mass $m_{\phi} \ll m$ that plays the role of a standard model field. The interaction is mediated by the heavy fermion $\chi$ with mass $M \gg$ $m, \mu^{2} / m$ and the same coupling to $A^{\mu}$ as $\psi$. The theory has a global $U(1)$ symmetry with charge +1 for $\phi^{*}, \psi_{1}^{*}$, and $\psi_{2},-1$ for $\phi, \psi_{2}^{*}$, and $\psi_{1}$, and 0 for $\chi$. Integrating out $\chi$, we obtain

$$
\mathcal{L}_{\text {int }}=\frac{h}{2}\left(\mathcal{O} \phi^{* 2}+\mathcal{O}^{*} \phi^{2}\right), \quad h \equiv \frac{2 \mu^{2}}{M} .
$$

The composite operator $\mathcal{O}$ defined in (2) has charge -2 under the global $U(1)$ symmetry. 
In a 4D unparticle theory, the interaction corresponding to (16) would typically be nonrenormalizable, becoming more important as the energy increases. That does not happen in our 2D toy model. But we can and will study the process in Fig. 1 in the unparticle limit and learn something about the transition region between the ordinary particle physics behavior at energies large compared to $m$ and the unparticle physics at low energies.

To that end, we consider the physical process $\phi+\phi \rightarrow$ Sommerfield stuff shown in Fig. 1: Because $\phi^{2}$ couples to $\mathcal{O}^{*}$, we can obtain the total cross section for this process from the discontinuity across the physical cut in the $\mathcal{O} 2$ point function. This is analogous to the optical theorem for ordinary particle production. For $\phi$ momenta $P_{1}$ and $P_{2}$, this is

$$
\sigma=\frac{\operatorname{Im} \mathcal{M}\left(P_{1}, P_{2} \rightarrow P_{1}, P_{2}\right)}{s},
$$

with $s=P^{2}, P=P_{1}+P_{2}$, and

$$
i \mathcal{M}\left(P_{1}, P_{2} \rightarrow P_{1}, P_{2}\right)=-i h^{2} \Delta_{\mathcal{O}}(P) .
$$

In the unparticle limit $(\sqrt{s} \ll m)$, using (13) or directly the phase space (14), we find the fractional power behavior expected with unparticle production:

$$
\sigma=\frac{A(a)}{2} \frac{h^{2}}{(\xi m)^{2 a}} \frac{1}{s^{1-a}} .
$$

On the other hand, in the free particle limit appropriate for high energies $\sqrt{s} \gg m$, we have $B(x) \rightarrow 1$ in (6), and then

$$
\sigma=\frac{h^{2}}{4} \frac{1}{s}
$$

which is the cross section for $\phi+\phi \rightarrow \bar{\psi}_{2}+\psi_{1}$.

Since we have the exact solution, we can study the transition between the two limits by writing (6) for arbitrary $x$ as

$$
\begin{aligned}
i \Delta_{\mathcal{O}}(x) & =i \Delta_{\mathcal{U}}(x) \exp [-4 \pi i a \Delta(x)] \\
& =i \Delta_{\mathcal{U}}(x) \sum_{n=0}^{\infty} \frac{(-4 \pi a)^{n}}{n !}[i \Delta(x)]^{n} .
\end{aligned}
$$

At distances not large compared to $1 / \mathrm{m}$, the higher terms in the sum in (21) become relevant. Notice that, in (21), we have expanded in $a$ only the terms involving the massive boson propagator. This is critical to our results. It would be a mistake to expand $i \Delta_{u}$ in powers of $a$. This would introduce spurious infrared divergences because the massless boson propagator is sick in $1+1$ dimensions [14]. The model describes not massive and massless bosons but rather massive bosons and unparticle stuff. In momentum space, we obtain

$$
\begin{aligned}
i \Delta_{\mathcal{O}}(P)= & \sum_{n=0}^{\infty} \frac{(-4 \pi a)^{n}}{n !} \int \frac{d^{2} p_{u}}{(2 \pi)^{2}} i \Delta u(p u) \\
& \times\left[\prod_{i=1}^{n} \frac{d^{2} p_{i}}{(2 \pi)^{2}} i \Delta\left(p_{i}\right)\right](2 \pi)^{2} \delta^{2}\left(P-p_{u}-\sum_{j=1}^{n} p_{j}\right) .
\end{aligned}
$$

This describes a sum of 2-point diagrams in which the incoming momentum $P$ splits between the unparticle propagator and $n$ massive scalar propagators. Each $\Delta$ is associated with the propagation of a free massive scalar field, so this gives the discontinuity

$$
\begin{aligned}
\Phi(P)= & \frac{A(a)}{(\xi m)^{2 a}} \sum_{n=0}^{\infty} \frac{(-4 \pi a)^{n}}{n !} \int \frac{d^{2} p u}{(2 \pi)^{2}} \theta\left(p_{u}^{0}\right) \theta\left(p_{u}^{2}\right)\left(p_{u}^{2}\right)^{a} \\
& \times\left[\prod_{i=1}^{n} \frac{d^{2} p_{i}}{(2 \pi)^{2}} 2 \pi \delta\left(p_{i}^{2}-m^{2}\right) \theta\left(p_{i}^{0}\right)\right] \\
& \times(2 \pi)^{2} \delta^{2}\left(P-p_{u}-\sum_{j=1}^{n} p_{j}\right) .
\end{aligned}
$$

For $\sqrt{s}<N m$, only the first $N$ terms in (23) (those involving the production of fewer than $N$ massive bosons) contribute, and (18) describes the production of unparticle stuff plus between 0 and $N-1$ massive bosons. For $\sqrt{s}<$ $m$, we have pure unparticle behavior. As we go to higher energies, the unparticle stuff is always present, but the emission of more and more massive bosons builds up the inclusive result for free-fermion production. This happens quickly if $a$ is small but very gradually for $a$ close to -1 .

One can easily obtain explicit results in the case of small $a$, when only the first few terms in the expansion contribute. The leading correction in $a$ comes from $n=1$ :

$$
\Phi^{(1)}=-a \theta(\sqrt{s}-m) \ln \frac{\sqrt{s}}{m}+\mathcal{O}\left(a^{2}\right)
$$

which gives the total phase space as

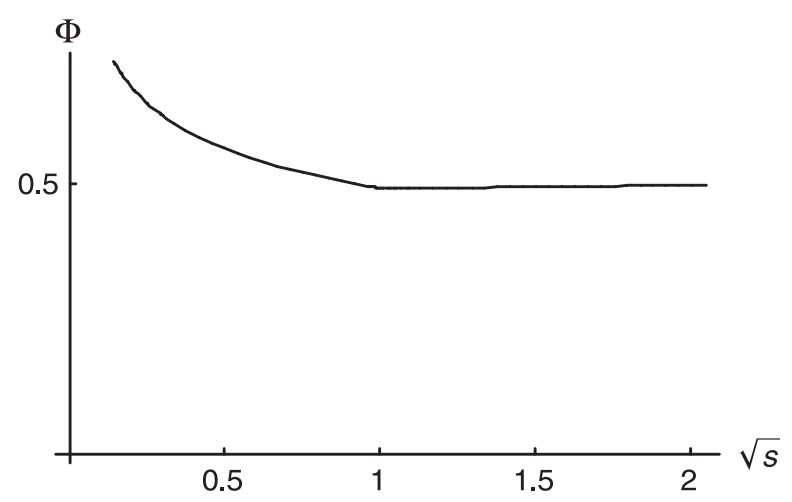

FIG. 2. Phase space $\Phi$ for the disappearance process in Fig. 1 as a function of the energy $\sqrt{s}$ (in units of $m$ ) for $a=-0.1$. 


$$
\Phi=\frac{1}{2}-a\left[\ln \left(\frac{2}{e^{\gamma_{E}}} \frac{\xi m}{\sqrt{s}}\right)+\theta(\sqrt{s}-m) \ln \frac{\sqrt{s}}{m}\right]+\mathcal{O}\left(a^{2}\right)
$$

For energies $\sqrt{s}>m$, this expression reduces to

$$
\Phi=\frac{1}{2}+\mathcal{O}\left(a^{2}\right),
$$

that is, the free-fermion result (20). Thus, for $|a| \ll 1$ there is a discontinuity in $d \Phi / d \sqrt{s}$ at $\sqrt{s}=m$, where a transition occurs from pure unparticle behavior below energy $m$ to pure free-fermion behavior above $m$ (see Fig. 2) [19]. To this order, the free-fermion behavior is a sum of the unparticle and the massive scalar contributions.

For larger values of $|a|$, higher powers of $a$ must be included in (25) to approximate the free-fermion regime. Since each new massive scalar gives a contribution with only one additional power of $a$, if $a$ is close to -1 , the freefermion behavior is approached very slowly. In fact, the limit $a \rightarrow-1$ is singular, and it corresponds to the Schwinger model $\left(m_{0}=0\right)$. As is often the case, it is not trivial to obtain a gauge theory as the limit of a theory with a massive vector boson. The unparticle stuff is absent since $A(-1)=0$, and the spectrum includes only a massive boson with $m^{2}=e^{2} / \pi$. The case of the Schwinger model has been studied in Refs. [20,21].

We find this picture of the unparticle scale $\Lambda_{u}=m$ in the Sommerfield model very satisfying. There is a close analog between the way $m$ enters in the process of Fig. 1 and the way the dimensional transmutation scale $\Lambda_{\mathrm{QCD}}$ enters in inclusive processes in QCD. In QCD, the physical states are hadrons, typically with masses of the order of $\Lambda_{\mathrm{QCD}}$ unless they are protected by some symmetry (like the pions). But in the total $e^{+} e^{-}$cross section into hadrons (to pick the simplest and most famous example) at high energy $E$, the sum over physical states reproduces the "parton model" result with calculable corrections of order $1 / \ln \left(E / \Lambda_{\mathrm{QCD}}\right)$. We have shown that the process of Fig. 1 in the Sommerfield model works the same way, with the physical states being the massive boson and unparticle stuff.

We are grateful to C. Cordova, V. Lysov, P. Petrov, A. Sajjad, and D. Simmons-Duffin for discussions. This research is supported in part by the National Science Foundation under Grant No. PHY-0244821. *georgi@physics.harvard.edu

+kats@physics.harvard.edu

[1] H. Georgi, Phys. Rev. Lett. 98, 221601 (2007).

[2] H. Georgi, Phys. Lett. B 650, 275 (2007).

[3] We prefer "unparticle stuff" to "unparticles" for the physical states, because it is not clear to us what the noun "unparticle" is supposed to mean and certainly not clear whether it should be singular or plural.

[4] T. Banks and A. Zaks, Nucl. Phys. B196, 189 (1982).

[5] Georgi's Ph.D. advisor and Schwinger's student.

[6] C. M. Sommerfield, Ann. Phys. (N.Y.) 26, 1 (1964).

[7] W.E. Thirring and J.E. Wess, Ann. Phys. (N.Y.) 27, 331 (1964).

[8] J. S. Schwinger, Phys. Rev. 128, 2425 (1962).

[9] We will use conventions in which $g^{\mu \nu}=\operatorname{diag}(1,-1)$ and $\gamma^{5}=\operatorname{diag}(1,-1)$

[10] $K_{0}$ is the modified Bessel function of the second kind, and $x_{0}$ is an arbitrary constant that will cancel out in the following. For $y \rightarrow \infty, K_{0}(y) \sim \sqrt{\pi / 2 y} e^{-y} \rightarrow 0$. For $y \rightarrow 0, \quad K_{0}(y)=-\ln (y / 2)-\gamma_{E}+\mathcal{O}\left(y^{2}\right)$, where $\gamma_{E}=$ $-\Gamma^{\prime}(1) \simeq 0.577$ is Euler's constant. Note that $\Delta(0)-$ $D(0)=(i / 2 \pi) \ln \left(e^{\gamma_{E}} x_{0} m / 2\right)$ is finite.

[11] H. Georgi and Y. Kats (to be published).

[12] There we will also include more complete references to the unparticle and Sommerfield model literature.

[13] Here and below, we incorporate a dimensional factor of $1 /(\xi m)^{2 a}$ in the unparticle propagator so it matches smoothly onto the $\mathcal{O}$ propagator.

[14] This statement has a long history in the mathematical physics literature, going back at least to Ref. [15]. For an early summary in English, see [16]. See also [17]. It is also worth noting that Ref. [15] introduces the notion of "infraparticles"- an approach to continuous mass representations of the Poincaré group, which of course includes unparticle stuff. See [18], where we learned of this interesting early reference.

[15] B. Schroer, Fortschr. Phys. 11, 1 (1963).

[16] J. Tarski, J. Math. Phys. (N.Y.) 5, 1713 (1964).

[17] S. R. Coleman, Commun. Math. Phys. 31, 259 (1973).

[18] B. Schroer, arXiv:0804.3563.

[19] The linear approximation (25) is not valid for $\sqrt{s} \ll m$ due to large $\ln \sqrt{s}$, but we have the exact expression (14).

[20] A. Casher, J. B. Kogut, and L. Susskind, Phys. Rev. Lett. 31, 792 (1973).

[21] A. Casher, J. B. Kogut, and L. Susskind, Phys. Rev. D 10, 732 (1974). 